\title{
MedienPädagogik
}

Zeitschrift für Theorie und Praxis der Medienbildung

www.medienpaed.com

ISSN 1424-3636

Jahrbuch Medienpädagogik 15:

Erziehungswissenschaftliche und medienpädagogische Online-Forschung:

Herausforderungen und Perspektiven

Herausgegeben von Johannes Fromme, Stefan Iske, Therese Leik, Steffi

Rehfeld, Jasmin Bastian, Manuela Pietraß und Klaus Rummler

\section{Materialität und digitale Medialität in der erziehungswissenschaftlichen Medienforschung}

\section{Ein praxeologisch-diskursanalytisch perspektivierter Vermittlungsversuch}

Patrick Bettinger

\section{Zusammenfassung}

Qualitative Sozialforschung steht vor methodischen und methodologischen Herausforderungen, da sich Sozialität im Kontext der Digitalität auf grundlegende Weise wandelt. Der Beitrag schlägt eine Perspektive vor, die sich der «medialen Verfasstheit) von Phänomenen widmet und hierbei die komplexen Verschränkungen von Menschen und Medien auf unterschiedlichen Ebenen berücksichtigt. Auf Grundlage einer praxeologisch-diskursanalytisch fundierten Herangehensweise wird gezeigt, wie Medialität und Materialität als aufeinander bezogene und quer zur Unterscheidung von Online- und Offline-Sphäre liegende Analysedimensionen erschlossen und medientheoretisch fruchtbar gemacht werden können, um die Konstitution von sozio-medialen Konstellationen zu erfassen. Die hier vorgeschlagene Herangehensweise wird am Bereich der qualitativen Medienbildungsforschung exemplarisch skizziert. Erste methodische Konkretisierungen werden im Zuge dessen mit Blick auf die Analyse diskursiver Praxis veranschaulicht. 


\title{
Materiality and Digital Mediality in Educational Media Research. A Mediation Attempt from the Perspective of Praxeology \& Discourse Analysis
}

\begin{abstract}
Qualitative social research in the context of digitality faces methodological and methodological challenges, as sociality is fundamentally changing. The paper proposes a perspective that addresses the (medial constitution〉 of phenomena, taking into account the complex entanglements of people and media at different levels. On the basis of a praxeological-discourseanalytical approach, it will be shown how mediality and materiality can be explored as interrelated analytical dimensions across the differentiation of online and offline spheres and made fruitful in terms of media theory to capture the constitution of socio-medial constellations. The approach proposed here is outlined as an example in the field of qualitative media education research. Methodological concretisations will be illustrated regarding the analysis of discursive practice.
\end{abstract}

\section{Einleitung und Problemaufriss - Digitalität und qualitative Forschung in der Erziehungswissen- schaft}

Der gegenwärtige medienpädagogische Diskurs um Digitalität, die als wesentliches Signum unserer gegenwärtigen Kultur verhandelt wird (Stalder 2017), legt nahe, dass es eines Medienbegriffs bedarf, der sich nicht auf technisch-instrumentelle Aspekte beschränkt und Medien nicht als die alleinige Nutzung von Endgeräten begreift. Stattdessen wird eine Perspektive eingefordert, die den medientechnologischen Wandel der Gegenwart als tiefgreifenden und umfassenden kulturellen Wandel auffasst, welcher massgeblich und auf mehreren Ebenen sowie in allen gesellschaftlichen Feldern entscheidend durch (aber nicht ausschliesslich) digitale Medien geprägt wird. So lässt sich - um dieses Problemfeld nur kurz anzudeuten 
- neben den Anforderungen im Umgang mit Hard- oder Software eine fundamentale (quasi infrastrukturelle) Ebene algorithmisierter Wirklichkeitskonstruktionen (Hepp 2016) erkennen, die derart in unseren Alltag eingeflochten ist, dass wir die hier wirksame, dem Software-Code folgende Logik, nicht bewusst als solche erfahren:

«Algorithmen haben ihre Logik in die Struktur aller sozialer Prozesse, Interaktionen und Erfahrungen eingewoben, deren Entfaltung von Rechenleistung abhängig ist» (Roberge und Seyfert 2017, 7).

Die 〈Invisibilität〉 der Medien kommt hier besonders deutlich zum Tragen - Medien, und besonders digitale Medien, verschwinden förmlich in ihrem Gebrauch (Krämer 2008, 28) bzw. bleiben durch routinisierte mediale Dekodierung und Konventionalisierung im Alltag zumeist intransparent (Genz und Gévaudan 2016, 62) - was ihrer Wirkmächtigkeit aber selbstverständlich keinen Abbruch tut.

Bereits diese erste Annäherung macht deutlich, dass die qualitative Forschung damit vor einem Problem steht: Wie soll etwas analysiert, interpretiert und rekonstruiert werden, das sich derart der Wahrnehmung entzieht und sich als verschachteltes Gefüge in alltägliche Lebenszusammenhänge eingefügt hat? Um diesen Veränderungsprozess in seiner Vielschichtigkeit (sinn-)verstehend rekonstruieren zu können, bedarf es nicht nur entsprechender theoretischer Weiterentwicklungen, sondern zugleich der Reflexion methodologischer und methodischer Aspekte. Nimmt man die in den letzten Jahren publizierten Arbeiten als Indikator, scheint die (deutschsprachige) qualitative Sozialforschung bislang eher zaghaft vorzugehen. Zwar finden sich punktuell Arbeiten, die einen Beitrag zu dieser Problemstellung liefern (Mikos und Wegener 2017; Schirmer, Sander und Wenninger 2015), von einer systematischen und umfassenden Weiterentwicklung qualitativer Ansätze vor dem Hintergrund des oben skizzierten Wandels kann aber noch keine Rede sein. 
Es zeichnet sich ab, dass in der (deutschsprachigen) qualitativen Sozialforschung hinsichtlich method(olog)ischer Innovationen mit Bezug auf den gegenwärtigen medienkulturellen Wandel scheinbar Zurückhaltung dominiert. Sozialität wird eher selten als grundlegend verändert verstanden, sondern nach wie vor auf Basis etablierter Herangehensweisen erforscht, d.h. mit Rekurs auf Grundlagentheorien (wie z.B. Symbolischer Interaktionismus, Phänomenologie), deren Wurzeln bis zum Anfang des 20. Jahrhunderts reichen. Medien werden im Anschluss an diese Traditionen bezüglich methodischer Fragen tendenziell als additiver Aspekt von sozialen Phänomenen begriffen oder gänzlich ausgeblendet. Noch weiter schränkt sich die Auswahl ein, wenn man nach dezidiert erziehungswissenschaftlichen Beiträgen sucht, die sich aus fachspezifischer Sicht mit methodologisch-methodischen Herausforderungen der Untersuchung von Erziehungs-, Lern-, Bildungs- oder Sozialisationsprozessen im Kontext des medienkulturellen Wandels befassen (als Ausnahme siehe die Beiträge in der "Forschungswerkstatt Medienpädagogik» von Knaus 2017; 2018). Wenngleich es einerseits nachvollziehbar ist, zunächst halbwegs sichere Fahrwasser aufzusuchen und qualitative Medienforschung vom bewährten erkenntnistheoretischen Terrain ausgehend zu betreiben, so sollte andererseits klar sein, dass die mehrere Jahrzehnte alten Grundlagenwerke, auf die sich die qualitative Sozialforschung stützt, zu einer Zeit verfasst wurden, in der die fundamentale Bedeutung digitaler Medialität für soziale Zusammenhänge nicht absehbar war. Insofern scheint es angemessen, grundlegende Fragen der Sozialforschung - wie etwa nach der Konstitution ihres Gegenstandes - teils (er)neu(t) zu stellen, mindestens aber weiterzudenken. Bezugnehmend auf dieses Grossprojekt blickt der vorliegende Beitrag auf einen Teilaspekt, nämlich die Frage, wie Materialität und digitale Medialität aus Sicht einer qualitativ ausgerichteten erziehungswissenschaftlichen Medienforschung konzeptionell gefasst und erschlossen werden können.

Hierzu nimmt dieser Beitrag eine Perspektive ein, die sich insbesondere auf die Verbindung von praxis- und diskurstheoretischen Ansätzen stützt. Es wird dargelegt, welche methodologischen 
Implikationen sich aus diesen Überlegungen für die qualitative Analyse sozio-medialer Phänomene ergeben. Hierzu wird zunächst gezeigt, welchen Stellenwert Materialität - als ein Aspekt digitaler Medien - für praxeologische Ansätze hat und inwiefern Materialität und Medialität unter einem praxistheoretischen Dach als aufeinander bezogene Konzepte gedacht werden können (Kap. 2). Daraufhin wird argumentiert, inwiefern der Einbezug einer diskurstheoretischen Perspektive - und letztlich einer Erweiterung des analytischen Konstrukts auf materiell-diskursive Praktiken - einen analytischen Gewinn bringen kann (Kap. 3). Anschliessend wird am Beispiel qualitativer Medienbildungsforschung ausschnitthaft demonstriert, wie sich ein solcher Ansatz auf einen konkreten Gegenstand beziehen lässt. Hierbei wird im Anschluss an die von Wrana dargelegte Analyse diskursiver Praktiken grob auf mögliche methodische Aspekte eingegangen (Kap. 4). Der Beitrag endet mit einer kritischen Reflexion der vorgeschlagenen Perspektive (Kap. 5).

\section{Materialität und Medialität aus praxeologischer Sicht}

Um zu der hier intendierten methodologischen Skizze hinzuführen, soll in einem ersten Schritt dargelegt werden, welche Implikationen sich aus einem praxistheoretischen Verständnis von Materialität und Medialität ergeben. Praxistheoretische Arbeiten ${ }^{1}$ (insbesondere der jüngeren Vergangenheit) betonen quasi durchgehend, dass materielle Artefakte wesentliche Bestandteile sozialer Praktiken sind (Hillebrandt 2014, 76ff.; Reckwitz 2014; Schmidt 2012, 62ff.; Wieser 2004). Oftmals wird im Zuge dieser Argumentationsgänge auf die

1 Ist nachfolgend die Rede von Praxistheorie, so geschieht dies im Bewusstsein über die Verschiedenheit praxistheoretischer Ansätze (siehe bspw. Schäfer 2016). Für den Kontext der hier verfolgten Argumentation werden diese Unterschiede vernachlässigt, da angenommen wird, dass sich zentrale Grundannahmen finden, die von allen Praxistheorien geteilt werden (Reckwitz 2003) und die allgemeine Rede von 〈den Praxistheorien〉 im Rahmen dieses Beitrags rechtfertigen. 
Akteur-Netzwerk-Theorie (ANT) (Latour 2007) verwiesen, die sich besonders dadurch auszeichnet, dass sie die genuine Handlungsmacht der Dinge als elementaren Bestandteil des Sozialen hervorhebt und den Blick konsequent auf unterschiedliche Prozesse und Qualitäten der Verbindung von menschlichen und nicht-menschlichen Grössen richtet. Die grundlegende Annahme der ANT bringt Latour wie folgt auf den Punkt: «Die Dinge machen etwas, sie sind nicht nur die Fläche oder die Projektoren unseres sozialen Lebens» (Latour 2001, 245). Materialität wäre im Verständnis der ANT demnach als Teil eines fluiden Prozesses zu verstehen, der durch Formen des Zusammenwirkens (temporär) verketteter Grössen bestimmt wird. Wieser $(2008,427)$ hält bezüglich der spezifischen Vorstellung von sozio-materiellen Verschränkungen der ANT fest: «Verschiedene Räume und Zeiten sind in Materialitäten und durch Ereignisse miteinander verbunden [Herv. i.O.]». Damit rücken Artefakte aus Sicht der sie konstituierenden Austauschprozesse in den Fokus. Mit Blick auf sozialtheoretische Anschlüsse wird die ANT einerseits teilweise selbst als Praxistheorie verstanden (Hillebrandt 2014, 78; Reckwitz 2003, 291). Andererseits wird auf ein theoretisches Defizit hingewiesen, das sich auf das simplizistische Verständnis von Handeln bei Latour bezieht (i.S.v. Handeln als Bewirken eines Unterschiedes), welchem eine praxeologische Alternative bzw. Erweiterung entgegen gestellt werden kann (Schäffer 2013). Hierdurch lassen sich unter anderem Konzepte wie Verstetigung und Beharrlichkeit in die von der Vorstellung prinzipiell fragiler Wirklichkeit ausgehenden ANT einflechten. Welcher Lesart der ANT man auch folgt, es zeigen sich zahlreiche 〈komplementaritätsverdächtige〉 Theorieelemente zwischen ANT und Praxistheorien. In dieser Hinsicht hält Wieser (2012) fest ${ }^{2}$ :

2 Trotz dieser Parallelen soll an dieser Stelle darauf hingewiesen werden, dass Wieser (2012) auch auf Unterschiede zwischen den Ansätzen hinweist: Mit Verweis auf den praxistheoretischen Ansatz von Schatzki zeigt er, dass z.B. die posthumanistische Position Latours durchaus als inkompatibel mit einer praxistheoretischen Idee der Einzigartigkeit menschlicher Handlungsträgerschaft aufgefasst werden kann. 
«Mit den Praxistheorien teilt die ANT die Kritik an kognitivistischen und intentionalistischen Verkürzungen gängiger Handlungstheorien als auch die Kritik an das [sic!] Handeln normierender und determinierender Strukturen. Gemeinsam ist ihnen eine Prozessperspektive auf soziales Handeln und Kultur. Beide 〈Theorien〉 problematisieren die Natur/KulturUnterscheidung und versuchen dem Antagonismus von Subjektivismus und Objektivismus zu entgehen» (ebd., 206).

Auf Grundlage der Berührungs- und Anschlusspunkte an die ANT scheint ein praxistheoretischer Zugang somit für die Erschliessung der Rolle von Materialität in der qualitativen Forschung passend. Medien können aus dieser Perspektive zunächst als «spezifisches Ensemble materialer Artefakte» (Reckwitz 2010, 163) aufgefasst werden, die allerdings eben nicht nur passiv mit Inhalt bespielt und als Instrumente dem menschlichen Willen unterworfen werden, sondern - folgt man Latour - einen aktiven Anteil an der sozialen Wirklichkeit haben, indem sie potenziell «ermächtigen, ermöglichen, anbieten, ermutigen, erlauben, nahelegen, beeinflussen, verhindern, autorisieren, ausschließen und so fort》 (Latour 2007, 124).

Wie verhält es sich nun mit dem hier verfolgten Anspruch, Medialität auf praxeologischer Basis zu denken? Neben der Möglichkeit, im Anschluss an Latour Artefakte als Bestandteile von Praktiken zu berücksichtigen, lassen sich auch Argumente finden, inwiefern die ANT gerade für die Erforschung digitaler Medialität Anregungen bietet. So kann mit Wieser (2012, 113f.) hervorgehoben werden, dass die ANT für eine praxeologisch fundierte Erforschung medienkultureller Phänomene vielversprechend ist, da sie konsequent die Prozesshaftigkeit von Phänomenen im Blick hat: «Sie [die ANT, PB] stellt die Performativität und Materialität sozialen Handelns sowie die Medialität von Technik heraus» (ebd., 114). Versuche, Medialität über einen starken Performativitätsbegriff zu fassen, finden sich auch vonseiten medientheoretischer Arbeiten. So weisen Genz und Gévaudan $(2016,61)$ darauf hin, dass es Medialität ohne Materialität 
nicht geben kann, da Zeichen immer auf bestimmte Formen der Materialisierung - also performative Akte - angewiesen sind. Materialität wird von den Autor*innen demnach als unterschiedliche Manifestationsformen von Zeichen im Prozess der Semiose gefasst. Insofern weist der Ansatz von Genz und Gévaudan durchaus eine gewisse Nähe zur ANT auf, die weder von einer strukturalistisch verstandenen Vorgängigkeit der Zeichen noch von einer passiv-inhaltsleeren Existenz von Materialität ausgeht, sondern materiellsemiotischen Netzwerken als assoziative Verkettungen einen konstitutiven Wert beimisst (Law 2009).

Vor dem Hintergrund des in diesem Beitrag verfolgten praxistheoretischen Zugangs bietet es sich (nicht zuletzt aus Gründen der Wahrung methodischer Anschlussfähigkeit) an, darüber hinaus an sozialsemiotische Konzepte anzuschliessen, die auf Praktiken des Zeichengebrauchs blicken und hierbei zeichenhafte Bedeutungsproduktion und -rezeption als soziale Prozesse begreifen (Meier 2014, 41). Medialität wird damit zu einer, sich performativ entfaltenden, Praxis des Zeichengebrauchs im Zusammenspiel mit bestimmten technologischen Materialisierungsoptionen. Performativität als wesentlichen Aspekt von Medialität zu begreifen entspricht auch der Annahme, dass Medien - und insbesondere digitale Medien - sich erst in ihrem Vollzug offenbaren und ihre Potenziale entfalten (bzw. deren Grenzen ersichtlich werden) (Münker 2013). Mit Leonardi (2010, o.S.) lässt sich dementsprechend festhalten:

«Especially in the case of digital artifacts, what may matter most about 'materiality〉 is that artifacts and their consequences are created and shaped through interaction».

In diese Richtung argumentiert auch Krämer (2004), die auf die konstitutive Verwobenheit von Performativität und Medialität hinweist. Performativität versteht sie als «eine Dimension aller kulturellen Praktiken im Spannungsverhältnis zwischen einem Ereignis und seiner Wahrnehmung» (ebd., 21) und weist auf die Nähe zur Position des «Mediengenerativismus» (ebd., 23) hin. Diese ist - im 
Anschluss an die Arbeiten von Flusser, Kittler und Virilio - dadurch gekennzeichnet, dass Medien nicht nur als übertragende Grössen betrachtet werden, sondern das Übertragene zugleich auch prägen. Rückt man in diesem Sinne also von einer essentialistischen Vorstellung (der Medien〉 als vergegenständlichte und neutrale Übermittler ab und richtet stattdessen den Blick auf Medialität als «übergreifende Form- und Strukturaspekte» (Jörissen 2014, 503), so muss die Frage nach den Aufforderungsstrukturen (den sogenannten Affordanzen) der Medien als sich im produktiven sowie rezeptiven Praxisvollzug entfaltende Grössen betrachtet werden. Medialität stellt sich damit als komplexes Interdependenzverhältnis aus sozialen, semiotischen und technisch-materiellen Grössen dar, die aufgrund ihres kokonstitutiven Charakters analytisch aufeinander bezogen werden müssen (Schüttpelz 2013). Digitale Medialität ist dann als relationales Konzept zu fassen, bei dem sich spezifische Hard- und Softwarekonstellationen sowie eine «Modellierung und parametrisierbare[n] Verwendung von Materialität innerhalb von Software» (Passoth 2017, 62) in unterschiedlicher Weise verknüpfen. Besonders letztere Eigenschaft, die Passoth (ebd.) als «Runtime» bezeichnet, fordert geradezu eine Perspektive ein, die digitale Medialität in actu erfasst, also deren performative Vollzugsdimension berücksichtigt. Die vorgeschlagene Herangehensweise impliziert damit eine Verschiebung der Perspektive: Anstelle der Frage nach der Materialität digitaler Medien tritt die Frage nach Prozessen und Formen der Materialisierung im Kontext digitaler Medialität in den Vordergrund. Digitale Medialität stellt in diesem Verständnis die Online/Offline-Dichotomie stark infrage, da das performative Verständnis des fokussierten Gegenstandsbereiches sich einer klaren Zuordnung zu einer Sphäre verwehrt. Vielmehr wäre die hier eingenommene Position zum Verhältnis von Materialität und Medialität als querliegend zu verstehen, da der daraus resultierende Blick auf (Re-)Formierung heterogener Konstellationen jenseits dichotomer Vorstellungen operiert und dezidiert eine multiple Relationalität vertritt. 


\section{Methodologische Annäherungsarenen: Prakti- ken und Diskurse als analytische Fluchtpunkte sozio-medialer Konstellationen}

Im Folgenden soll dargestellt werden, inwiefern ein diskurstheoretisch ausgerichteter Blick geeignet erscheint, den Zusammenhang von Materialität und Medialität näher zu beleuchten. Grundsätzlich schliesst die Argumentation hierzu an einen Vorwurf an, der den Praxistheorien von diskursanalytischer Seite entgegengebracht werden kann: Mit Reckwitz (2008, 196ff.) weisen praxeologische Zugänge hinsichtlich ihrer methodologischen Ausrichtung ein Problem auf, wenn es um die Frage geht, wie die explizit beobachtbaren Handlungen oder Äusserungen mit dem impliziten Wissen zusammenhängen, welches in den Praxistheorien als handlungsleitend gilt. Hiermit ergibt sich für Reckwitz das Problem der Zugänglichkeit des Impliziten: «Der Forscher ist immer auf einen «Rückschluss〉 vom Expliziten aufs Implizite, von den Bewegungen auf den (sozialen Sinn〉 angewiesen» (ebd., 196). Besonders problematisch wird dies, wenn es um die Erforschung von nicht unmittelbar beobachtbaren, zeitlich zurückliegenden Praktiken geht - hier scheint kaum ein Weg an methodischen Erweiterungen vorbeizuführen, wie etwa dem Einbezug von Praxisbeschreibungen, Egodokumenten oder anderen Artefakten in die Analyse. Mit dieser Erweiterung rückt die praxeologische Analyse in die Nähe der Diskursforschung, da dieses Vorgehen die «impliziten Wissensordnungen einer historischen Phase» (ebd., 201) in den Blick nimmt, was dem diskursanalytischen Vorgehen sehr nahe kommt. ${ }^{3}$

Ein weiterer Aspekt spricht für eine diskursanalytische Erweiterung der im vorangegangenen Kapitel dargelegten Position: Latour hegt hinsichtlich der ANT offenbar kein Interesse an einem Konzept von (Kontext), sondern betrachtet die Welt ausschliesslich als unterschiedliche, mehr oder weniger miteinander verbundene Netzwerke, wie Wieser $(2012,208)$ kritisch anmerkt. Diese Annahme

3 Zur Kritik an dieser Position siehe Volbers (2015). 
findet weder in praxis- noch aus diskurstheoretischen Arbeiten eine Entsprechung. Beide Strömungen beziehen, wenngleich auf unterschiedliche Art und Weise, gesellschaftliche Aspekte in ihre Analysen mit ein und erkennen grundsätzlich überindividuelle Macht- und Herrschaftsstrukturen als Aspekte der sozialen Welt an. An dieser Stelle wird vorgeschlagen, das Problem der ausbleibenden Berücksichtigung 〈situationsübergreifender) Mechanismen bei der ANT über einen Einbezug einer diskursanalytischen Perspektive einzuholen. Die Frage nach der (Diskursivität von Artefakten〉 erweist sich als ein im diskurstheoretischen Zusammenhang bislang nur ansatzweise bearbeiteter Komplex (vgl. z.B. van Dyk et al. 2014). In ihrer prägnanten Analyse der Positionen Latours und Foucaults legt van Dyk (2010, 188f.) dar, dass eine Stärke der ANT in der Sensibilisierung für unterschiedliche Formen von Vermittlung besteht, die besonders vor dem Hintergrund eines Einbezugs von Fragen ungleicher Existenzbedingungen im Anschluss an Foucault ausbaufähig erscheint. Sie weist zudem darauf hin, dass «die zurecht monierte Vernachlässigung der (vielfältigen) Nutzungsweisen von Artefakten sowie die Vernachlässigung von inkorporierter (habitualisierter) zugunsten vergegenständlichter, objektivierter Geschichte (praxis-)theoretisch einzufangen [wäre]» (ebd., 189). Die im Rahmen des Beitrags vorgeschlagene Bezugnahme auf Materialität diskursiver Praxis soll in eben diese genannte Lücke stossen und einen ersten Impuls bieten, wie diesem Desiderat beizukommen wäre.

Neben der von Reckwitz (2008, 201ff.) vorgeschlagenen Analyse von Praxis/Diskurs-Formationen als eine Verbindung von Praxisund Diskursanalyse, erweist sich das Konzept der Analyse diskursiver Praxis von Wrana (2012a, 2012b, 2015) als vielversprechender Ankerpunkt. Diskursivität wird hierbei im Anschluss an Foucault als "Handlungsweisen, in denen sich das Sagbare und Sichtbare formt und in denen die Bedeutungen und Gegenstände des Wissens ebenso konstituiert werden wie die Subjektpositionen der diskursiv Handelnden» (Wrana 2012b, 196), sowie als Handlungsweisen, «in denen Wahrheit als Relationierung von Bedeutungsfeldern, 
Wissensobjekten und Subjektivitäten performativ hergestellt wird» (ebd.), verstanden. Es geht dann im Anschluss an die dargelegte Anforderung, Materialität einzubeziehen, darum, den analytischen Fokus auf materiell-diskursive Praktiken ${ }^{4}$ von Hybridakteuren zu richten, die aus den dynamischen Verflechtungen von menschlichen und nicht-menschlichen Grössen hervorgehen. Wenn van Dyk $(2010,191)$ vorschlägt, "zwischen den unterschiedlichen Formen und Ausprägungen des Diskursiven zu unterschieden» und sich hierbei nicht auf sprachliche Äusserungen zu beschränken, so kann über die Analyse der materiell-diskursiven Praktiken als Formen einer aus heterogenen Netzwerken emergierenden agency eine erste Antwort gegeben werden. Praxis ist damit nicht als exklusives menschliches Tätigsein zu verstehen, sondern als ein aus der Verknüpfung menschlicher und nicht-menschlicher Elemente hervorgehender Prozess, in dem allerdings nicht - wie Latour meint - von einer vollkommenen Voraussetzungslosigkeit ausgegangen werden kann, sondern durchaus situationsübergreifende Kräfte am Werk sind, deren Wirkmächtigkeit über die diskursanalytische Perspektive einzuholen wäre.

Bezugspunkt der Analyse bilden dann diskursive Schemata ${ }^{5}$, die in diesen Praktiken zur Geltung kommen, aber auch - im Sinne unterschiedlicher Aggregatzustände - in digitalen Artefakten etwa als Code oder Hardwarekonfigurationen materialisiert sind und hier in Form von Affordanzen (sozusagen vorsprachlich) auf Diskursfiguren verweisen können. Die Affordanzen medialer Artefakte werden damit sowohl als diskursiv geprägt wie auch diskursiv prägend erkennbar und sind dementsprechend nur in ihrer jeweiligen Eingebundenheit in relationale Gefüge verstehbar. Im Aufeinandertreffen unterschiedlich inkorporierter bzw. materialisierter diskursiver Schemata entfalten sich - diskursanalytisch betrachtet - je spezifische Formen der Bedeutungszuschreibung bzw. -umschreibung, (Re-)Positionierung oder Positionszuschreibung und schliesslich

4 Ausführlicher und mit spezifischem epistemologischen Einschlag zum Begriff der materiell-diskursiven Praktiken siehe Barad (2012) bzw. kritisch dazu Keller (2017).

5 Siehe dazu auch Bettinger (2016). 
Formen der (Re-)Produktion von Subjektivität, wobei die (In-)Stabilität und Transformation von hybriden Konstellationen in den Blick geraten. Gleichzeitig können - um eine die ANT ergänzende Perspektive vorzuschlagen (van Dyk 2010, 189) - in Form sedimentierter diskursiver Schemata Verknüpfungen relative Stabilität erhalten. Mit Dölemeyer und Rodatz $(2010,215)$ kann damit von einer Analyse des «sozialen Lebens von Wissen〉 in Form von dynamischen, materialisierten Verbindungen gesprochen werden. Der Blick richtet sich auf die performative Kraft von Handlungsprogrammen bzw. dem Aufeinandertreffen mit menschlichem Handeln sowie deren diskursive Verwicklungen.

Affinitäten eines solchen Zugangs zeigen sich auch hinsichtlich des zuvor dargelegten sozialsemiotischen Verständnisses von Medialität. Durch die vorgeschlagene Perspektive geraten gesellschaftliche Kontexte (hier verstanden als Wissensfelder) in den Blick, die den Zeichengebrauch bspw. in Form von Konventionen regulieren und prästrukturieren (selbstverständlich ohne diesen zu determinieren), gleichzeitig aber wiederum durch (Zeichen-)Praxis überhaupt erst hervorgebracht werden. Meier (2014) hält für den damit verbundenen, ideologiekritischen Impetus dieses Verständnisses der Sozialsemiotik fest:

«Er [der Ideologiebegriff, P.B.] lenkt die Aufmerksamkeit auf das Vorhandensein von Dominanzverhältnissen in gesellschaftlichen Formationen, die dazu führen, dass Interessen und Weltsichten bestimmter dominant verorteter Akteure, Gruppen, Ethnien, Kulturen und Diskursen (massenmedial unterstützt) stärkeren Einfluss in der kollektiven Wirklichkeitskonstruktion haben als andere» (ebd., 43).

Dass an dieser Stelle Bezüge zu diskursanalytischen Vorgehensweisen auf der Hand liegen, ist evident. Auch bei Genz und Gévaudan (2016, 162ff.) finden sich, im Zusammenhang mit der Frage nach der Entstehung und Veränderung von Konventionen des Mediengebrauchs, Bezüge zur Bedeutsamkeit eines Einbezugs eines 
diskursanalytischen (bzw. dispositivanalytischen) Zugangs. Im Sinne einer vorläufigen Arbeitsdefinition im Anschluss an die bislang entfalteten Positionen kann Medialität damit als diskursiv kontextualisiertes und bedeutungs(re)produzierendes Relationierungsgeschehen bestimmt werden, das sich im Rahmen bestimmter (technologischer und semiotischer) Materialisierungsmöglichkeiten entfaltet. Wie ein solcher Zugang an einem konkreten medienpädagogischen Gegenstandsbereich zur Anwendung gebracht werden kann, wird nachfolgend am Beispiel der Medienbildungsforschung veranschaulicht.

\section{Die Materialität und Medialität diskursiver Pra- xis am Beispiel der qualitativen Medienbildungs- forschung}

Abschliessend soll zumindest kurz darauf eingegangen werden, wie die entworfene Perspektive im Bereich der qualitativen Medienbildungsforschung ihr Potenzial entfalten kann. Versteht man dieses Forschungsfeld in der Tradition der transformatorischen Bildungstheorie und -forschung (Koller 1999; Marotzki 1990; Nohl 2006; Rosenberg 2011), welche die prozessuale Entfaltung sich verändernder Selbst- und Weltverhältnisse fokussiert, so ergeben sich neue theoretische und empirische Möglichkeiten aber auch Anforderungen. Ausgehend von einer Abkehr von anthropozentrisch gedachter Subjektivität (Bettinger 2017) muss Medienbildungsforschung als Analyse materiell-diskursiver Praxis sich über eine Neukonzeption sowohl des theoretischen Grundgerüsts als auch des empirischen Zugriffs Gedanken machen. Während Bildung als Transformation von Selbst- und Weltverhältnissen eng mit der Biographieforschung verbunden ist und auf ein dementsprechendes, individuell-reflexiv ausgerichtetes Subjektverständnis zurückgreift (siehe bspw. Schulze 2006), erfordert die hier vorgeschlagene Ausrichtung auf materielldiskursive Praxis, Bildung konsequent als relationale Prozessgenese (Bettinger 2018, 379ff.; Brauckmann 2015) zu erfassen. Der für die transformatorische Bildungstheorie zentrale Dualismus von Selbst 
und Weltverhältnissen scheint angesichts dieser Herangehensweise kaum haltbar und bedarf zumindest aber einer grundlegenden Modifikation. Eng damit verbunden ist die Frage, was genau sich in Bildungsprozessen denn eigentlich transformiert. Den bspw. von Koller (2012, 99ff.) vorgeschlagenen Theorieoptionen wäre eine Alternative hinzuzufügen, die Bildung «auf der Ebene der Transformation subjektivierender Relationierungen» (Jörissen 2015, 228) von menschlichen und nicht-menschlichen Akteuren verortet. Der medienpädagogische Gegenstandsbereich der Medienbildungsforschung erscheint dann als Schauplatz verteilter agency zwischen menschlichen und nicht-menschlichen Akteuren (Rammert 2006) und es rücken Formen und Ausprägungen von Machtverhältnissen in den Vordergrund, die Subjektivierungsprozesse prägen indem sie etwa in den verschiedensten Materialisierungsformen Sichtund Sagbarkeiten regulieren. Bildung im Kontext der Digitalität wird so als «Produktive Verwicklung» (Allert und Asmussen 2017) verstanden, bei der die subjektivierende Kraft sozio-technischer Arrangements im Mittelpunkt steht. Medienbildungsforschung blickt damit verstärkt auf potenzielle Konstellationen und Formen von Subversion und Widerständigkeit gegen hegemoniale oder gar unterdrückte Subjektfiguren oder aber Formen des Aufbegehrens gegen ebensolche, wobei die Herstellung neuer Verknüpfungen von besonderem Interesse ist. Fragen nach dem «Nicht-Gesagten〉 stehen hier ebenso im Fokus wie Fragen nach dem (Nicht-Materialisierten). Medienbildungsforschung als Analyse materiell-diskursiver Praktiken zu betreiben heisst zudem, normativ-reflektierte Fragen nach wünschenswerten und nicht-wünschenswerten Prozessen der (In-) Stabilisierung heterogener Netzwerke zu stellen.

Nicht zuletzt aufgrund der Nähe dieser Überlegungen zu poststrukturalistischen Theorien ist eine vom konkreten Gegenstand abgelöste Darstellung methodischer Schritte zwar kaum möglich, gleichwohl lassen sich - um zumindest eine erste Orientierung zu geben - grobe methodische Prinzipien und Werkzeuge benennen: Ein forschungspraktischer Zugriff im Anschluss an die hier skizzierten 
methodologischen Eckpunkte richtet sich darauf, wie diskursive Schemata Formen und Prozesse der Verknüpfung (bzw. Auflösung von Verknüpfungen) in relationalen Gefügen bedingen oder anders herum, wie sich diskursive Schemata im Zuge von Bildungsprozessen transformieren, wie sich in diesen Prozessen agency auf unterschiedliche Entitäten verteilt und wie in diesen Prozessen Medialität zum Ausdruck kommt. Hierbei ist ein triangulativ angelegtes Forschungsdesign naheliegend ${ }^{6}$, dass unterschiedliche Datensorten einbezieht, insbesondere wenn es - wie im Fall rekonstruktiver (Medien-)Bildungsforschung - darum geht, bereits in der Vergangenheit liegende Prozessverläufe zu analysieren. Da der Königsweg der Beobachtung von Praktiken hier im Regelfall nicht umsetzbar ist, können Kombinationen aus Interview- und Artefaktanalysen, die sich unter anderem - etwa im Falle digitaler Artefakte - auf archivierte Artefaktversionen stützen, eine Möglichkeit darstellen, um sich bereits vergangenen konstellationalen Veränderungen anzunähern. Entscheidend scheint (mehr denn je), den Status der herangezogenen Daten sorgfältig vor dem Hintergrund ihrer relationalen Einbettung in den Phänomenzusammenhang zu reflektieren und ebenso das methodische Vorgehen an Gegenstand und Fragestellung anzupassen. Auf Grundlage eines Diskursverständnisses, das sich der Unterscheidung von sprachlich vs. nicht-sprachlich bzw. diskursiv und nicht-diskursiv entzieht und die unterschiedlichen Manifestationsformen von Diskursen berücksichtigt (Wrana und Langer 2007), wären in einem ersten Schritt ausführliche Beschreibungen der sich (potenziell) verändernden bzw. veränderten sozio-medialen Konstellationen ausgehend von den Formen der Verknüpfung der unterschiedlichen Elemente zu leisten, deren Schwerpunktsetzung wiederum abhängig von der zugrunde gelegten Forschungsfrage ist. ${ }^{7}$ Hierzu bedarf es eines möglichst präzisen Vokabulars, das

6 Kritisch zur Einschätzung des Potenzials von Triangulation in der qualitativen Forschung siehe z. B. Wrana (2011).

7 Auch wenn sie methodologisch anders gelagert ist als die hier eingenommene Position, bietet die Situationsanalyse nach Clarke (2012) hier sehr gute Anregungen, nicht zuletzt was die Möglichkeiten der Visualisierung im Forschungsprozess betrifft. 
der Spezifik des Gegenstandes gerecht wird (siehe hierzu bspw. Rammert 2006) und sowohl dessen figurative als auch prozedurale und positionale Aspekte berücksichtigt (Wrana 2015, 121) und gleichzeitig der Einschränkung Rechnung trägt, dass die Konstellationen niemals abschliessend dargestellt werden können. Leitend kann hier die Frage sein, was im Vollzug von materiell-diskursiven Praktiken - bzw. in der sprachlichen Darstellung des (potenziell in der Vergangenheit liegenden) Vollzugs dieser Praktiken - auf eine bestimmte Art und Weise relationiert wird bzw. wurde (ebd., 130) sowie «auf welche Weise Akteure an einer Praxis partizipieren [oder partizipierten, PB] und mit welchen Ein- und Ausschlüssen sie zu Partizipanden einer Praxis werden [bzw. wurden, PB]» (Wrana 2012b, 203). Die im analytischen Vorgehen leitende Perspektive zielt auf die Rekonstruktion des im Praxisvollzug hergestellten Verhältnisses von Aussage und Äusserungsakt ab, die die jeweiligen Gegenstände in ihrem Zusammenspiel in Form unterschiedlicher «figuraler Operationen» (Wrana 2015, 135) konstituieren. Hierbei würden im Falle der hier vorgeschlagenen Analyse sozio-medialer Verflechtungen nicht-sprachliche Praktiken noch wesentlich stärker in den Fokus rücken, als dies etwa bei den von Maier Reinhard, Ryter Krebs und Wrana (2012) oder Wrana (2015) herangezogenen Beispielen der Fall ist, die deutlich auf verbalsprachliche Äusserungen bezogen sind. Die Schwierigkeit besteht dann darin, eine angemessene und handhabbare methodische «Übersetzungsleistung〉 solcher nicht-sprachlich verfasster Praktiken zu gewähren.

Für den Bereich der Medienbildungsforschung sind insbesondere prozessrekonstruktive Fragen von Interesse. Eine Möglichkeit, diesem Problem forschungsmethodisch zu begegnen, besteht in der fallinternen Kontrastierung der Veränderung der rekonstruierten relationalen Gefüge unter Einbezug archivierter Daten bzw. Artefaktversionen (etwa von Websites), an denen sich erste Ansatzpunkte vergangener Konstellationen zeigen können (Bettinger 2018, 151ff.). Bei der Arbeit mit (bspw. biographischen) Interviews kommt es in dieser Hinsicht umso mehr darauf an, den Status von Aussagen 
in Bezug auf ihre Verweishaftigkeit zu prüfen und über die Rekonstruktion von Bezügen zu den veränderten Artefaktversionen die in der Vergangenheit liegenden relationalen Verhältnisse methodisch in den Griff zu bekommen. Problematisch erscheint hier, dass Aussagen über vergangene Praktiken nicht mit den tatsächlichen Praktiken gleichgesetzt werden können. ${ }^{8}$ Der Status von Erzählungen erweist sich hierbei als methodisch prekärer Anknüpfungspunkt. Gerade an diesem Punkt können weitere Datensorten, die über die verbalsprachlichen Erzählungen hinaus gehen, wertvolle Einsichten liefern. Über Einbezug der genannten (medialen) Artefakte bzw. deren Veränderungsgeschichte und der Suche nach homologen 〈Spuren〉 in Artefakten und Interviews, lässt sich versuchen, die Rekonstruktion von in der Vergangenheit liegenden Konstellationen methodisch besser abzusichern. Abgesehen von einigen wenigen Ansätzen, die sich der qualitativen Analyse von Artefakten widmen (z. B. Lueger und Froschauer 2018; Nohl 2013), ist in diesem Bereich jedoch noch viel Pionierarbeit zu leisten.

\section{Fazit}

Der vorliegende Text hatte das Ziel dazu beizutragen, die erziehungswissenschaftliche Medienforschung für ihren sich im permanenten Wandel befindlichen Phänomenbereich zu sensibilisieren und den Horizont möglicher Fragen und Forschungsperspektiven zumindest ein klein wenig zu erweitern. Wie gezeigt wurde, sensibilisiert ein praxistheoretischer Zugang dafür, die Qualität der Einbettung in bzw. Beteiligung von Medien an alltäglichen Handlungsvollzügen zu erkennen. Denn neben der Möglichkeit eines geschmeidigen und fast unbemerkten Einfügens in den Alltag, kann 〈Medienpraxis〉 auch mit Widerständigkeit einhergehen und Irritationen bedingen. Diese Schärfung des Blicks für die Mikroebene des Umgangs mit Medien und der Sensibilisierung für Interdependenzen scheinen

8 Hier sei auf den seit vielen Jahren andauernden Disput in Bezug auf den Zusammenhang von Erzählung und Erfahrung hingewiesen, der methodologisch insbesondere mit Blick auf biographisches Material unterschiedlich bearbeitet wird (siehe hierzu bspw. Kauppert 2010). 
besonders aus erziehungswissenschaftlicher Sicht bedeutsam, denn sie zielen auf die Frage ab, wie sich im Umgang mit Medien in Lern, Bildungs- oder Sozialisationsprozessen auf unterschiedliche Art Prägekräfte entfalten, die sowohl wünschenswerte als auch problematische Konstellationen bedingen können. Durch die Verbindung mit einer diskursanalytischen Perspektive geraten darüber hinaus überindividuelle Aspekte digitaler Medialität in den Blick, wie etwa ungleichheitsbedingende Macht- und Herrschaftsaspekte, die Verteilung und Ausprägung von Sprecher*innenpositionen in bestimmten Diskursen sowie überhaupt die Frage der diskursiven Bezüge, die sich in den Praktiken zeigen. Hierdurch ergibt sich die Möglichkeit, die medienpädagogische Forschung mit neuen Fragestellungen anzureichern, die bislang wenig berücksichtigt wurden. Konsequent weitergedacht läuft ein solches Projekt darauf hinaus, die Frage des Gegenstandes der Medienpädagogik neu zu stellen. Sozio-mediale Konstellationen zu untersuchen bedeutet letztlich, die implizite anthropozentrische Färbung von Verfahren der qualitativen Sozialforschung, die sich aus ihrer grundlagentheoretischen Ausrichtung ergibt, kritisch zu reflektieren und - wo angemessen - zu modifizieren. Hierbei werden die komplexen Verschränkungen in den Blick genommen, die über dichotome Unterscheidungen wie Subjekt/Objekt oder Online/Offline hinaus gehen und stattdessen dem performativen Charakter hybrider Verflechtungen Rechnung tragen.

Neben dem Potenzial der hier skizzierten Perspektive müssen Schwierigkeiten eines solchen Zugangs eingeräumt werden. Offensichtlich ist der grosse methodologische und methodische Aufwand, der einem stimmigen Forschungsdesign abverlangt wird, das sich auf die oben dargelegten Positionen bezieht. Die hier nur knapp dargestellten Verbindungen zwischen praxis- und diskursanalytischen Positionen dürften bei näherer Betrachtung durchaus erkenntnistheoretisches Reibungspotenzial aufweisen, das sich unter anderem hinsichtlich teils unterschiedlicher subjekttheoretischer Ausgangspunkte sowie dem Status verschiedener Wissensformen abzeichnet. Insbesondere ist hier der Einbezug der ANT zu nennen, welcher 
hier im Sinne einer Heuristik zu verstehen ist und von einer eng an Latour ausgerichteten Lesart abweicht, wobei dementsprechend dessen analytische Prämissen infrage gestellt werden. Trotz dieser Schwierigkeiten scheint es geboten, den Versuch zu wagen, digitaler Medialität und Materialität auf Grundlage des vorgeschlagenen Entwurfs zu begegnen, um die Vielschichtigkeit zumindest ansatzweise zu durchdringen und ein besseres Verständnis der sich im Wandel befindenden Welt zu bekommen, was letztendlich ein Kernanliegen der Medienpädagogik darstellt.

\section{Literatur}

Allert, Heidrun, und Michael Asmussen. 2017. "Bildung als produktive Verwicklung». In Digitalität und Selbst. Interdisziplinäre Perspektiven auf Subjektivierungs- und Bildungsprozesse, herausgegeben von Heidrun Allert und Michael Asmussen, 27-68. Bielefeld: transcript.

Barad, Karen. 2012. Agentieller Realismus. Über die Bedeutung materielldiskursiver Praktiken. Berlin: Suhrkamp.

Bettinger, Patrick. 2016. «Mediale Diskurse und biographische Transformationen». In Mediale Diskurse, Kampagnen, Öffentlichkeiten, herausgegeben von Johannes Fromme, Florian Kiefer, und Jens Holze, 9-33. Wiesbaden: Springer Fachmedien. https://doi.org/10.1007/978-3-65810526-6_2.

Bettinger, Patrick. 2017. "Hybride Subjektivität(en) in mediatisierten Welten als Bezugspunkte der erziehungswissenschaftlichen Medienforschung». MerzWissenschaft, 7-18.

Bettinger, Patrick. 2018. Praxeologische Medienbildung. Theoretische und empirische Perspektiven auf sozio-mediale Habitustransformationen. Wiesbaden: Springer VS. https://doi.org/10.1007/978-3-658-21849-2.

Brauckmann, Bianca. 2015. «Lernen und Bildung in relationaler Perspektive. Ein Gedankenexperiment». In Subjekt Medium Bildung, herausgegeben von Benjamin Jörissen und Torsten Meyer, 191-213. Wiesbaden: Springer Fachmedien. https://doi.org/10.1007/978-3-658-06171-5_10.

Clarke, Adele E. 2012. Situationsanalyse: Grounded Theory nach dem Postmodern Turn. Wiesbaden: Springer VS. 
Dölemeyer, Anne, und Mathias Rodatz. 2010. «Diskurse und die Welt der Ameisen. Foucault mit Latour lesen (und umgekehrt)». In Zwischen Sprachspiel und Methode. Perspektiven der Diskursanalyse, herausgegeben von Robert Feustel und Maximilian Schochow, 197-220. Bielefeld: transcript.

Genz, Julia, und Paul Gévaudan. 2016. Medialität, Materialität, Kodierung. Grundzüge einer allgemeinen Theorie der Medien. Bielefeld: transcript.

Hepp, Andreas. 2016. «Kommunikations- und Medienwissenschaft in datengetriebenen Zeiten». Publizistik 61 (3): 225-46. https://doi. org/10.1007/s11616-016-0263-y.

Hillebrandt, Frank. 2014. Soziologische Praxistheorien. Eine Einführung. Wiesbaden: Springer VS. https://doi.org/10.1007/978-3-531-94097-7.

Jörissen, Benjamin. 2014. «Digitale Medialität». In Handbuch Pädagogische Anthropologie, herausgegeben von Christoph Wulf und Jörg Zirfas, 503-13. Wiesbaden: Springer Fachmedien. https://doi. org/10.1007/978-3-531-18970-3_46.

Jörissen, Benjamin. 2015. «Bildung der Dinge: Design und Subjektivation». In Subjekt Medium Bildung, herausgegeben von Benjamin Jörissen und Torsten Meyer, 215-33. Wiesbaden: Springer Fachmedien. https://doi.org/10.1007/978-3-658-06171-5_11.

Kauppert, Michael. 2010. Erfahrung und Erzählung. Zur Topologie des Wissens. 2. Aufl. Wiesbaden: Springer VS. https://doi.org/10.1007/978-3531-92308-6.

Keller, Rainer. 2017. «Neuer Materialismus und Neuer Spiritualismus? Diskursforschung und die Herausforderung der Materialitäten». Österreichische Zeitschrift für Volkskunde LXXXI (120): 5-31.

Knaus, Thomas, Hrsg. 2017. Forschungswerkstatt Medienpädagogik: Projekt - Theorie - Methode. Bd. 1. München: kopaed.

Knaus, Thomas, Hrsg. 2018. Forschungswerkstatt Medienpädagogik: Projekt - Theorie - Methode. Bd. 2. München: kopaed.

Koller, Hans-Christoph. 1999. Bildung und Widerstreit: zur Struktur biographischer Bildungsprozesse in der (Post-)Moderne. München: Wilhelm Fink.

Koller, Hans-Christoph. 2012. Bildung anders denken. Einführung in die Theorie transformatorischer Bildungsprozesse. Stuttgart: Kohlhammer.

Krämer, Sibylle. 2004. «Was haben 〈Performativität〉 und 〈Medialität〉 miteinander zu tun? Plädoyer für eine in der〈Aisthetisierung〉 gründende Konzeption des Performativen». In Medialität und Performativität, herausgegeben von Sibylle Krämer, 13-32. München: W. Fink. 
Krämer, Sibylle. 2008. Medium, Bote, Übertragung. Kleine Metaphysik der Medialität. Frankfurt am Main: Suhrkamp.

Latour, Bruno. 2001. «Eine Soziologie ohne Objekt? Anmerkungen zur Interobjektivität». Berliner Journal für Soziologie 11 (2): 237-52. https:// doi.org/10.1007/BF03204016.

Latour, Bruno. 2007. Eine neue Soziologie für eine neue Gesellschaft. Einführung in die Akteur-Netzwerk-Theorie. Frankfurt am Main: Suhrkamp.

Law, John. 2009. «Actor Network Theory and Material Semiotics». In The new Blackwell companion to social theory, herausgegeben von Bryan S. Turner. Chichester, West Sussex, United Kingdom; Malden, MA, USA: Wiley-Blackwell.

Leonardi, Paul M. 2010. «Digital Materiality? How Artifacts without Matter, Matter». First Monday 15 (6). https://doi.org/10.5210/fm.v15i6.3036.

Lueger, Manfred, und Ulrike Froschauer. 2018. Artefaktanalyse. Grundlagen und Verfahren. Wiesbaden: Springer VS. https://doi. org/10.1007/978-3-658-18907-5.

Maier Reinhard, Christiane, Barbara Ryter Krebs, und Daniel Wrana. 2012. «Lesarten im Professionalisierungsprozess. Eine empirische Analyse der Verstehensprozesse in Lernberatungsgesprächen». In Professionalisierung in Lernberatungsgesprächen, herausgegeben von Daniel Wrana und Christiane Maier Reinhard. Opladen: Budrich.

Marotzki, Winfried. 1990. Entwurf einer strukturalen Bildungstheorie. Biographietheoretische Auslegung von Bildungsprozessen in hochkomplexen Gesellschaften. Weinheim: Deutscher Studienverlag.

Meier, Stefan. 2014. Visuelle Stile. Zur Sozialsemiotik visueller Medienkultur und konvergenter Design-Praxis. Bielefeld: transcript.

Mikos, Lothar, und Claudia Wegener, Hrsg. 2017. Qualitative Medienforschung. Ein Handbuch. 2. Aufl. Konstanz \& München: UVK.

Münker, Stefan. 2013. "Media in use: how the practice shapes the mediality of media». Distinktion: Scandinavian Journal of Social Theory 14: 246-53.

Nohl, Arnd-Michael. 2006. Bildung und Spontaneität. Phasen biographischer Wandlungsprozesse in drei Lebensaltern - Empirische Rekonstruktionen und pragmatistische Reflexionen. Opladen: Budrich.

Nohl, Arnd-Michael. 2013. "Sozialisation in konjunktiven, organisierten und institutionalisierten Transaktionsräumen: Zum Aufwachsen mit materiellen Artefakten». Zeitschrift für Erziehungswissenschaft 16 (2): 189-202. 
Passoth, Jan-Hendrik. 2017. «Hardware, Software, Runtime. Das Politische der (zumindest) dreifachen Materialität des Digitalen». Behemoth 10 (1): 57-73.

Rammert, Werner. 2006. «Technik in Aktion: Verteiltes Handeln in soziotechnischen Konstellationen». In Technografie: zur Mikrosoziologie der Technik, herausgegeben von Werner Rammert und Cornelius Schubert, 163-95. Frankfurt a.M. \& New York: Campus Verlag.

Reckwitz, Andreas. 2003. "Grundelemente einer Theorie sozialer Praktiken. Eine sozialtheoretische Perspektive». Zeitschrift für Soziologie 32 (4): 282-301.

Reckwitz, Andreas. 2008. "Praktiken und Diskurse. Eine sozialtheoretische und methodologische Relation». In Theoretische Empirie. Zur Relevanz qualitativer Forschung, herausgegeben von Herbert Kalthoff, Stefan Hirschauer, und Gesa Lindemann, 188-209. Frankfurt am Main: Suhrkamp.

Reckwitz, Andreas. 2010. Unscharfe Grenzen. Perspektiven der Kultursoziologie. 2. Aufl. Bielefeld: transcript.

Reckwitz, Andreas. 2014. «Die Materialisierung der Kultur». In Praxeologie: Beiträge zur interdisziplinären Reichweite praxistheoretischer Ansätze in den Geistes- und Sozialwissenschaften, herausgegeben von Friederike Elias, Albrecht Franz, Henning Murmann, und Ulrich W. Weiser, 13-25. Berlin; Boston: De Gruyter.

Roberge, Jonathan, und Robert Seyfert. 2017. «Was sind Algorithmuskulturen?» In Algorithmuskulturen. Über die rechnerische Konstruktion der Wirklichkeit, herausgegeben von Robert Seyfert und Jonathan Roberge, 7-40. Bielefeld: transcript.

Rosenberg, Florian v. 2011. Bildung und Habitustransformation. Empirische Rekonstruktionen und bildungstheoretische Reflexionen. Bielefeld: Transcript.

Schäfer, Hilmar. 2016. Praxistheorie. Ein soziologisches Forschungsprogramm. Bielefeld: transcript.

Schäffer, Burkhard. 2013. «〈Kontagion» mit dem Technischen. Zur dokumentarischen Interpretation der generationenspezifischen Einbindung in die Welt medientechnischer Dinge». In Die dokumentarische Methode und ihre Forschungspraxis. Grundlagen qualitativer Sozialforschung, herausgegeben von Ralf Bohnsack, Iris Nentwig-Gesemann, und Arnd-Michael Nohl, 3. Aufl., 51-74. Wiesbaden: VS Verlag für Sozialwissenschaften. 
Schirmer, Dominique, Nadine Sander, und Andreas Wenninger, Hrsg. 2015. Die qualitative Analyse internetbasierter Daten. Methodische Herausforderungen und Potenziale von Online-Medien. Wiesbaden: VS Verlag für Sozialwissenschaften. https://doi.org/10.1007/978-3-65806296-5.

Schmidt, Robert. 2012. Soziologie der Praktiken. Konzeptionelle Studien und empirische Analysen. Berlin: Suhrkamp.

Schulze, Theodor. 2006. «Biographieforschung in der Erziehungswissenschaft - Gegenstandbereich und Bedeutung». In Handbuch erziehungswissenschaftliche Biographieforschung, herausgegeben von Heinz-Hermann Krüger und Winfried Marotzki, 2. Aufl., 35-57. Wiesbaden: VS Verlag für Sozialwissenschaften. https://doi. org/10.1007/978-3-531-90010-0_3.

Schüttpelz, Erhard. 2013. «Elemente einer Akteur-Medien-Theorie». In Akteur-Medien-Theorie, herausgegeben von Tristan Thielmann und Erhard Schüttpelz, 9-67. Bielefeld: transcript.

Stalder, Felix. 2017. Kultur der Digitalität. 2. Aufl. Berlin: Suhrkamp.

van Dyk, Silke. 2010. «Verknüpfte Welt oder Foucault meets Latour. Zum Dispositiv als Assoziation». In Zwischen Sprachspiel und Methode. Perspektiven der Diskursanalyse, herausgegeben von Robert Feustel und Maximilian Schochow, 169-96. Bielefeld: transcript. https://doi. org/10.14361/transcript.9783839414293.169.

van Dyk, Silke, Antje Langer, Felicitas Macgilchrist, Daniel Wrana, und Alexander Ziem. 2014. "Discourse and beyond? Zum Verhältnis von Sprache, Materialität und Praxis». In Diskursforschung. Ein interdisziplinäres Handbuch, herausgegeben von Johannes Angermuller, Martin Nonhoff, Eva Herschinger, Felicitas Macgilchrist, Martin Reisigl, Juliette Wedl, Daniel Wrana, und Alexander Ziem, 347-63. Bielefeld: transcript. https://doi.org/10.14361/transcript.9783839427224.347.

Volbers, Jörg. 2015. "Theorie und Praxis im Pragmatismus und in der Praxistheorie». In Praxis denken. Konzepte und Kritik, herausgegeben von Thomas Alkemeyer, Volker Schürmann, und Jörg Volbers, 193-214. Wiesbaden: Springer VS. https://doi.org/10.1007/978-3-65808744-9_9.

Wieser, Matthias. 2004. «Inmitten der Dinge. Zum Verhältnis von sozialen Praktiken und Artefakten». In Doing Culture. Neue Positionen zum Verhältnis von Kultur und sozialer Praxis, herausgegeben von Karl H. Hörning und Julia Reuter, 92-107. Bielefeld: transcript. https://doi. org/10.14361/9783839402436-006. 
Wieser, Matthias. 2008. «Technik/Artefakte. Mattering Matter». In Poststrukturalistische Sozialwissenschaften, herausgegeben von Stephan Moebius und Andreas Reckwitz, 419-32. Frankfurt am Main: Suhrkamp.

Wieser, Matthias J. 2012. Das Netzwerk von Bruno Latour. Die Akteur-Netzwerk-Theorie zwischen Science \& Technology Studies und poststrukturalistischer Soziologie. Bielefeld: transcript.

Wrana, Daniel. 2011. «Zur Rekonstellation von Methoden in Forschungsstrategien». In Methodentriangulation in der qualitativen Bildungsforschung, herausgegeben von Jutta Ecarius und Ingrid Miethe, 207-24. Opladen, Berlin \& Farmington Hills: Budrich.

Wrana, Daniel. 2012a. «Diesseits von Diskursen und Praktiken. Methodologische Bemerkungen zu einem Verhältnis». In Feld und Theorie. Herausforderungen erziehungswissenschaftlicher Ethnographie, herausgegeben von Barbara Friebertshäuser, Helga Kelle, Heike Boller, Sabine Bollig, Christina Huf, Antje Langer, Marion Ott, und Sophia Richter, 185-200. Opladen, Berlin \& Toronto: Budrich.

Wrana, Daniel. 2012b. «Theoretische und methodologische Grundlagen der Analyse diskursiver Praktiken». In Professionalisierung in Lernberatungsgesprächen. Theoretische Grundlegungen und Empirische Untersuchungen, herausgegeben von Daniel Wrana und Christiane Maier Reinhard, 3:195-214. Opladen, Berlin \& Toronto: Budrich.

Wrana, Daniel. 2015. «Zur Methodik einer Analyse diskursiver Praktiken». In Methoden einer Soziologie der Praxis, herausgegeben von Franka Schäfer, Anna Daniel, und Frank Hillebrandt, 121-43. Bielefeld: transcript. https://doi.org/10.14361/9783839427163-005.

Wrana, Daniel, und Antje Langer. 2007. «An den Rändern der Diskurse. Jenseits der Unterscheidung diskursiver und nicht-diskursiver Praktiken». Forum Qualitative Sozialforschung 8 (2). https://doi. org/10.17169/FQS-8.2.253. 